\title{
SHAPIRO'S UNCERTAINTY PRINCIPLE RELATED TO THE WINDOWED FOURIER TRANSFORM ASSOCIATED WITH THE RIEMANN-LIOUVILLE OPERATOR
}

\author{
AYMEN HAMMAMI
}

Abstract. Quantitative Shapiro's dispersion uncertainty principle and umbrella theorem are proved for the windowed Fourier transform associated with the Riemann-Liouville operator.

Mathematics subject classification (2010): 42A38, 44A35.

Keywords and phrases: The windowed Fourier transform, uncertainty principle, Hilbert-Schmidt operator, time-frequency concentration, Fourier transform, dispersion principle, Shapiro's theorem, Umbrella theorem.

\section{REFERENCES}

[1] C. Baccar, N. B. Hamadi And L. T. RAChDi, Inversion formulas for the Riemann-Liouville transform and its dual associated with singular partial differential operators, Int. J. Math. Math. Sci., 2006 (2006) 1-26.

[2] C. BACCAR AND L. T. RAChDI, Spaces of $D_{L^{p}}$-type and a convolution product associated with the Riemann-Liouville operator, Bull. Math. Anal. Appl., vol. 1, issue 3 (2009) 16-41.

[3] A. Beurling, The collected works of Arne Beurling, Birkhäuser., vol. 1-2, Boston 1989.

[4] A. Bonami, B. Demange, P. Jaming, Hermite functions and uncertainty principles for the Fourier and the windowed Fourier transforms, Rev. Mat. Iberoam. 19 (2003), 23-55.

[5] W. Czaja, G. Gigante, Continuous Gabor transform for stronghypergroups, J. Fourier Anal Appl. 2003; 9: 321-339.

[6] A. ERDÉLYI, Asymptotic expansions, Dover publications, New-York 1956.

[7] D. GABOR, Theory of communication, J. Inst Elec Engr. 1946; 93: 429-441.

[8] S. Ghobber, S. OmRI, Time-frequency concentration of the windowed Hankel transform, Integral Transforms Spec Funct. 2014; 25: 481-496.

[9] S. GhobBer, Phase space localization of orthonormal sequences in $L_{\alpha}^{2}\left(\mathbb{R}_{+}\right)$, J. Approx. Theory 189: 123-136, 2015.

[10] S. GHobBer, Time-frequency concentration and localization operators in the Dunkl setting, J. Pseudo-Differ. Oper. Appl., 7 (3): 431-449, 2016.

[11] K. H. GRÖCHENIG, Foundations of time-frequency analysis, Birkhauser, Boston, 2001.

[12] P. JAMing, A. M. Powell, Uncertainty principles for orthonormal sequences, J. Funct. Anal. 243, (2007), 611-630.

[13] H. LAmouchi AND S. OMRI, Time-frequency localization for the short time Fourier transform, Integral Transforms Spec. Funct., 27 (1): 43-54, 2016.

[14] N. N. Lebedev, Special Functions and Their Applications, Dover publications, New-York 1972.

[15] E. Malinnikova, Orthonormal sequences in $L^{2}\left(\mathbb{R}^{d}\right)$ and time frequency localization, J. Fourier Anal. Appl. 16, (2010), 983-1006.

[16] S. OMRI AND L. T. RACHDI, An $L^{p}-L^{q}$ version of Morgan's theorem associated with RiemannLiouville transform, Int. J. Math. Anal., vol. 1, no. 17 (2007) 805-824.

[17] S. OMRI AND L. T. RACHDI, Heisenberg-Pauli-Weyl uncertainty principle for the Riemann-Liouville Operator, J. Inequal. Pure and Appl. Math., 9 (2008), Iss. 3, Art 88.

[18] L. T. RAChDi AND A. RouZ, On the range of the Fourier transform connected with RiemannLiouville operator, Ann. Math. Blaise Pascal, vol. 16, no. 2 (2009) 355-397. 
[19] S. SAITOH, Theory of reproducing kernels and its applications, Harlown: Longman Higher Education; 1988.

[20] H. S. SHAPIRO, Uncertainty principles for bases in $L^{2}(\mathbb{R})$, in: Proceedings of the Conference on Harmonic Analysis and Number Theory, CIRM, Marseille-Luminy, October 16-21 (2005).

[21] E. M. Stein, Interpolation of linear operators, Trans. Am. Math. Soc. 83, 482-492 (1956).

[22] K. TRIMÈche, Transformation intégrale de Weyl et théorème de Paley-Wiener associés à un opérateur différentiel singulier sur $(0,+\infty)$, J. Math. Pures Appl., 60 (1981) 51-98.

[23] K. TRIMÈCHE, Inversion of the Lions translation operator using genaralized wavelets, Appl. Comput. Harmon. Anal., 4 (1997) 97-112.

[24] M. W. Wong, Wavelet transforms and localization operators, Birkhäuser (2002). 